\title{
DEFLECTIONS OF THE VERTICAL AND GEOID HEIGHTS FROM GRAVITY GRADIENTS
}

\author{
$L$ VÖLGYESI ${ }^{* 1,2}$
}

[Manuscript received January 25, 2005]

\begin{abstract}
In the 20th century more than 60000 torsion balance measurements were made in Hungary. At present efforts are made to rescue the historical torsion balance data; today 24544 torsion balance measurements are available for further processing in computer database. Previously only the horizontal gradients of gravity were used by geophysicists, but there is a good possibility in geodesy to interpolate deflections of the vertical, and to compute geoid heights from curvature gradients of gravity.

First the theory of the interpolation method is discussed, than results of test computations are presented. We have selected a test area where all kind of torsion balance measurements are available at 249 points. There were 3 astrogeodetic points providing initial data for the interpolation, and 10 checkpoints for controlling the results. The size of our test area is about $750 \mathrm{~km}^{2}$ and the average site distance of torsion balance data is $1.5-2 \mathrm{~km}$. The standard deviations of geoid height and deflection of the vertical differences at checkpoints were about $\pm 1-3 \mathrm{~cm}$, and \pm 0.6 " respectively; which confirm that torsion balance measurements give good possibility to compute very precise deflections of the vertical and local geoid heights at least for flat areas.
\end{abstract}

Keywords: Geoid determination, deflection of the vertical, Torsion balance measurements, curvature gradients of gravity, inversion.

\section{Fundamentals of the interpolation method}

Let us consider distribution of deflections of the vertical in a small area where torsion balance measurements are available. Let computations be referred to a Cartesian system, having an arbitrary point $P_{0}$ within the examined area as origin. Let $+x$ and $+y$ be the axes of the system point to the north and to the east, respectively, and let axis $z$ coincide with vertical direction at $P_{0}$ so that its positive branch points downwards.

Thereby, direction $z$ at any point $P_{i}$ of the concerned area is parallel to the $z$-axis through point $P_{0}$, and the direction $x_{i}$ to the tangent of astronomical meridian through point $P_{0}$, as illustrated by the arbitrary point $P_{i}$ in Fig. 1 . The $z$-axis at point $P_{i}$ being parallel to the vertical at origin $P_{0}$, presumably, direction of vector $g_{i}$ at point $P_{i}$ does not coincide with direction $z$. In Fig.1. vector $\overline{P_{i} V}$ is, in fact, projection of vector $g_{i}$ on plane $x z$, while vector $\overline{P_{i} H}$ is projection of component $g_{x i}$ of vector $g_{i}$ on the same plane. (There are negligible differences between vectors $\overline{P_{i} V}$ and $g_{i}$ as well as $\overline{P_{i} H}$ and $g_{x i}$ ).

Be $\Phi_{0}$ the astronomical latitude of point $P_{0}$, and let $\Delta \Phi_{i}$ symbolize the angle between direction $P_{i} V$ and $\mathrm{z}$ at point $P_{i}$, so the astronomical latitude of point $P_{i}$ is:

$$
\Phi_{i}=\Phi_{0}+\Delta \Phi_{i} .
$$

While, according to Fig.1: $-g_{x i}=g_{i} \sin \Delta \Phi_{i}$; it is to be written, for a small angle $\Delta \Phi_{i}$, as:

$$
\Delta \Phi_{i}=-\frac{g_{x i}}{g_{i}} .
$$

The same can be written for the variation of astronomic longitude in plane $y z$ to:

\footnotetext{
${ }^{* 1}$ Budapest University of Technology end Economics, Department of Geodesy and Surveying

${ }^{* 2}$ Research Group of Physical Geodesy and Geodynamics of the Hungarian Academy of Sciences,

H-1521 Budapest, Hungary, Müegyetem rkp.3. E-mail:volgyesi@eik.bme.hu
} 


$$
\Delta \Lambda_{i} \cos \Phi_{i}=\frac{g_{y i}}{g_{i}} .
$$

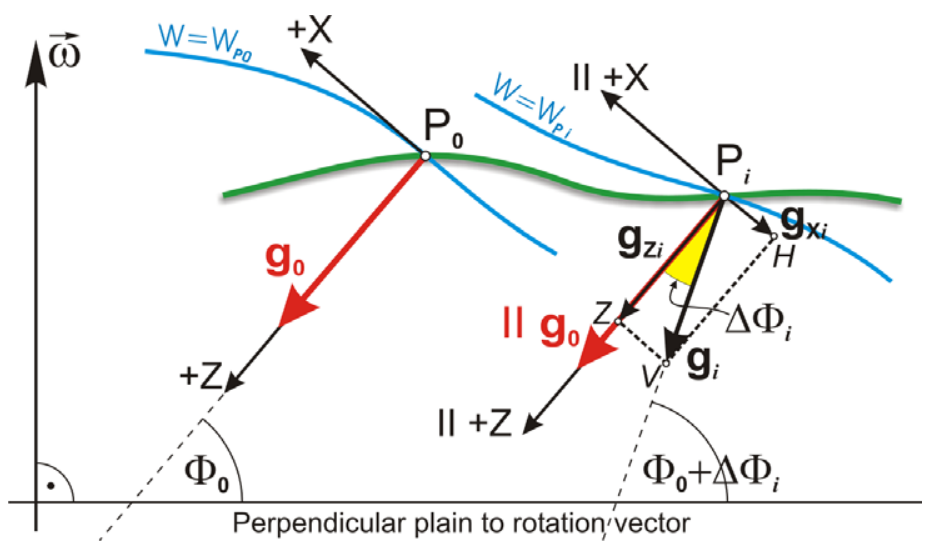

Fig. 1. Coordinate system for the interpolation

Equations (1) and (2) yield components $N$ and $E$ of the angle between geoid normal at points $P_{0}$ and $P_{i}$. Values $\Delta \Phi_{k}$ and $\Delta \Lambda_{k}$ for $P_{0}$ and some $P_{k}$ may be determined in a similar way. These may be applied for writing differences between $P_{i}$ and $P_{k}$ :

$$
\left(\Delta \Phi_{k}-\Delta \Phi_{i}\right)=-\left(\frac{g_{x k}}{g_{k}}-\frac{g_{x i}}{g_{i}}\right)
$$

and

$$
\left(\Delta \Lambda_{k}-\Delta \Lambda_{i}\right) \cos \widetilde{\Phi}=-\left(\frac{g_{y k}}{g_{k}}-\frac{g_{y i}}{g_{i}}\right)
$$

where $\widetilde{\Phi}$ is the mean value of astronomical latitude between points $P_{i}$ and $P_{k}$. By analogy with (1) and (2), (3) and (4) yield components $N$ and $E$ of the angle included by level surface normal at $P_{i}$ and $P_{k}$.

By introducing notations $g_{x}=\frac{\partial W}{\partial x}=W_{x}$ and $g_{y}=\frac{\partial W}{\partial y}=W_{y}(W$ is the potential of Earth's real gravity field), Eqs (3) and (4) may be written as:

$$
\left(\Delta \Phi_{k}-\Delta \Phi_{i}\right)=-\left(\frac{W_{x k}}{g_{k}}-\frac{W_{x i}}{g_{i}}\right)
$$

and

$$
\left(\Delta \Lambda_{k}-\Delta \Lambda_{i}\right) \cos \widetilde{\Phi}=-\left(\frac{W_{y k}}{g_{k}}-\frac{W_{y i}}{g_{i}}\right)
$$

respectively.

Level surfaces of the potential of normal gravity field, normal gravity, and directions of normal gravity vectors, in this relation, geodetic latitude and longitude of any point, termed normal geodetic latitude ${ }_{n} \varphi$ and normal geodetic longitude ${ }_{n} \lambda$, may be interpreted on the analogy of the Earth's real gravity field.

Relationships similar to (5) and (6) may be written between the variation of the gravity field direction in normal gravity field, that is, of normal geodetic coordinates ${ }_{n} \varphi$ and ${ }_{n} \lambda$ of points $P_{i}$ and $P_{k}$, and the derivatives conform to potential of the normal gravity field (normal potential): 


$$
\Delta_{n} \varphi_{k}-\Delta_{n} \varphi_{i}=-\left(\frac{U_{x k}}{\gamma_{k}}-\frac{U_{x i}}{\gamma_{i}}\right)
$$

and

$$
\left(\Delta_{n} \lambda_{k}-\Delta_{n} \lambda_{i}\right) \cos \tilde{\varphi}=-\left(\frac{U_{y k}}{\gamma_{k}}-\frac{U_{y i}}{\gamma_{i}}\right)
$$

where $U$ is the normal potential, and $\gamma_{i}, \gamma_{k}$ are the values of normal gravity at points $P_{i}$ and $P_{k}$. Let us subtract Eqs (5) and (7), as well as (6) and (8) from each other:

$$
\begin{gathered}
\left(\Delta \Phi_{k}-\Delta_{n} \varphi_{k}\right)-\left(\Delta \Phi_{i}-\Delta_{n} \varphi_{i}\right)=-\left(\frac{W_{x k}}{g_{k}}-\frac{W_{x i}}{g_{i}}\right)+\left(\frac{U_{x k}}{\gamma_{k}}-\frac{U_{x i}}{\gamma_{i}}\right) \\
{\left[\left(\Delta \Lambda_{k}-\Delta_{n} \lambda_{k}\right)-\left(\Delta \Lambda_{i}-\Delta_{n} \lambda_{i}\right)\right] \cos \tilde{\varphi}=-\left(\frac{W_{y k}}{g_{k}}-\frac{W_{y i}}{g_{i}}\right)+\left(\frac{U_{y k}}{\gamma_{k}}-\frac{U_{y i}}{\gamma_{i}}\right) .}
\end{gathered}
$$

By definition, differences (9) and (10) between astronomic and normal geodetic latitudes and longitudes yield differences of components $\xi$ and $\eta$ of deflection of the vertical between points $P_{i}$ and $P_{k}$ :

$$
\begin{aligned}
& \xi_{k}-\xi_{i}=-\left(\frac{W_{x k}}{g_{k}}-\frac{U_{x k}}{\gamma_{k}}\right)+\left(\frac{W_{x i}}{g_{i}}-\frac{U_{x i}}{\gamma_{i}}\right) \\
& \eta_{k}-\eta_{i}=-\left(\frac{W_{y k}}{g_{k}}-\frac{U_{y k}}{\gamma_{k}}\right)+\left(\frac{W_{y i}}{g_{i}}-\frac{U_{y i}}{\gamma_{i}}\right) .
\end{aligned}
$$

Inside a limited area of size $0.5^{\circ} \times 0.5^{\circ}$, approximations $\tilde{\gamma}=\widetilde{g}$ and $\widetilde{\Phi}={ }_{n} \widetilde{\varphi}=\widetilde{\varphi}$ are permissible - and so are single values $\tilde{g}$ and $\tilde{\varphi}$ valid for all the area rather than between two neighboring points alone (Selényi 1953, Badekas and Mueller 1967) to be indicated simply by $g$ and $\varphi$. Introducing notations

leads to equations:

$$
\Delta W=W-U
$$

$$
\begin{aligned}
& g\left(\xi_{k}-\xi_{i}\right)=-\Delta W_{x k}+\Delta W_{x i}, \\
& g\left(\eta_{k}-\eta_{i}\right)=-\Delta W_{y k}+\Delta W_{y i}
\end{aligned}
$$

Remind that in classic geodesy, deflection of the vertical is frequently interpreted as:

$$
\begin{gathered}
\xi=\Phi-\varphi \\
\eta=(\Lambda-\lambda) \cos \varphi
\end{gathered}
$$

where $\Phi$ and $\Lambda$ are astronomic coordinates, while $\varphi$ and $\lambda$ are geodetic (ellipsoidal) coordinates of point.

By physically interpreting the ellipsoid, serving as reference surface, as one level surface of the normal gravity field, than ellipsoidal and normal geodetic coordinates are related as $\varphi={ }_{n} \varphi-\kappa$ and $\lambda={ }_{n} \lambda$ ( $\kappa$ is the difference of directions of the normal gravity field between point $P$ on the earth surface and the ellipsoid surface along the normal plumb line at point $P$ ). The mentioned $0.5^{\circ} x$ $0.5^{\circ}$ area, variation of $\kappa$ is practically negligible (Magnitzki and Brovar 1964). Hence, (11) and (12) are also valid for the classical geodetic interpretation of deflection of the vertical. Thus, in the 
following, when interpreting of deflection of the vertical it is needless to distinguish between the two conceptions, permitting to use the concept of deflection of the vertical in both interpretations.

Components of deflections of the vertical - more closely, their values multiplied by $g$, that is, horizontal components - seemed to be determined by first derivatives of the potential. While torsion balance measurements yield second derivatives

$$
W_{\Delta}=\frac{\partial^{2} W}{\partial y^{2}}-\frac{\partial^{2} W}{\partial x^{2}} \quad \text { and } \quad W_{x y}=\frac{\partial^{2} W}{\partial x \partial y} .
$$

Thus, the computation problem is essentially an integration to be solved by approximation.

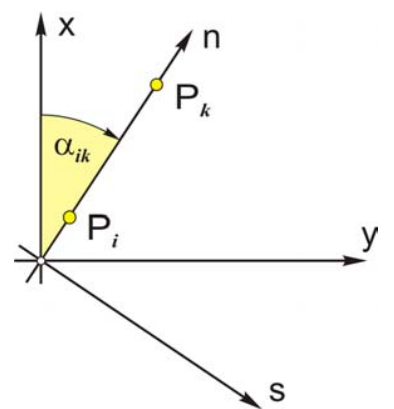

Fig. 2. $x, y \rightarrow n, s$ coordinate transformation

To this aim, first the coordinate transformation in Fig.2 will be performed, according to

Accordingly:

$$
\left[\begin{array}{c}
n \\
s
\end{array}\right]=\left[\begin{array}{cc}
\cos \alpha_{i k} & \sin \alpha_{i k} \\
-\sin \alpha_{i k} & \cos \alpha_{i k}
\end{array}\right]\left[\begin{array}{l}
x \\
y
\end{array}\right]
$$

$$
\begin{aligned}
& W_{n}=\frac{\partial W}{\partial n}=\frac{\partial W}{\partial x} \frac{\partial x}{\partial n}+\frac{\partial W}{\partial y} \frac{\partial y}{\partial n}=W_{x} \cos \alpha_{i k}+W_{y} \sin \alpha_{i k} \\
& W_{s}=\frac{\partial W}{\partial s}=\frac{\partial W}{\partial x} \frac{\partial x}{\partial s}+\frac{\partial W}{\partial y} \frac{\partial y}{\partial s}=-W_{x} \sin \alpha_{i k}+W_{y} \cos \alpha_{i k}
\end{aligned}
$$

while the second derivative:

$$
\frac{\partial^{2} W}{\partial n \partial s}=\frac{\partial^{2} W}{\partial x \partial y} \cos 2 \alpha_{i k}+\frac{1}{2}\left(\frac{\partial^{2} W}{\partial y^{2}}-\frac{\partial^{2} W}{\partial x^{2}}\right) \sin 2 \alpha_{i k}
$$

This latter $W_{n s}=\frac{\partial^{2} W}{\partial n \partial s}$ seems to result from torsion balance measurements, with the knowledge of azimuth $\alpha_{i k}$ of the direction connecting the two points examined.

Now, by integrating the left-hand side of (17) between limits $n_{i}$ and $n_{k}$ :

$$
\int_{n_{i}}^{n_{k}} \frac{\partial^{2} W}{\partial n \partial s} d n=\left(\frac{\partial W}{\partial s}\right)_{k}-\left(\frac{\partial W}{\partial s}\right)_{i}=W_{s k}-W_{s i}
$$

If points $P_{i}$ and $P_{k}$ are close enough to let variation of second derivative $W_{n s}$ be considered as linear, then integral (18) may be computed by trapezoid integral approximation formula:

$$
\int_{n_{i}}^{n_{k}} \frac{\partial^{2} W}{\partial n \partial s} d n=\frac{1}{2}\left[\left(\frac{\partial^{2} W}{\partial n \partial s}\right)_{i}+\left(\frac{\partial^{2} W}{\partial n \partial s}\right)_{k}\right]\left(n_{k}-n_{i}\right)=\frac{1}{2}\left[\left(W_{n s}\right)_{i}+\left(W_{n s}\right)_{k}\right] n_{i k}
$$


where $n_{i k}=n_{k}-n_{i}$ is the distance between points $P_{i}$ and $P_{k}$. On the other hand, by applying transformation (16), integral (18) yields:

$$
W_{s k}-W_{s i}=-\left(W_{x k}-W_{x i}\right) \sin \alpha_{i k}+\left(W_{y k}-W_{y i}\right) \cos \alpha_{i k} .
$$

Similar expression can be written for the potential $U$ of normal gravity field:

$$
U_{s k}-U_{s i}=-\left(U_{x k}-U_{x i}\right) \sin \alpha_{i k}+\left(U_{y k}-U_{y i}\right) \cos \alpha_{i k} .
$$

Subtracting (21) from (20) yields variation $G_{i k}=g \Delta \vartheta_{i k}$ of horizontal force component between points $P_{i}$ and $P_{k}$ in direction $n$. By taking (13) into consideration:

$$
G_{i k}=\left(-\Delta W_{x k}+\Delta W_{x i}\right) \sin \alpha_{i k}-\left(-\Delta W_{y k}+\Delta W_{y i}\right) \cos \alpha_{i k}
$$

after substituting (14) and (15):

$$
G_{i k}=g\left(\vartheta_{k}-\vartheta_{i}\right) \vartheta_{i k}=g\left(\xi_{k}-\xi_{i}\right) \sin \alpha_{i k}-g\left(\eta_{k}-\eta_{i}\right) \cos \alpha_{i k}
$$

The left-hand side of (22) may be computed by using (19). When using notation (13):

$$
G_{i k}=\frac{1}{2}\left[\left(\Delta W_{n s}\right)_{i}+\left(\Delta W_{n s}\right)_{k}\right] n_{i k}
$$

with $\Delta W_{n s}$ to be computed from (17):

$$
\Delta W_{n s}=\Delta W_{\Delta} \sin 2 \alpha_{i k}+\Delta W_{x y} \cos 2 \alpha_{i k}
$$

where $\Delta W_{\Delta}=W_{\Delta}-U_{\Delta}$ and $\Delta W_{x y}=W_{x y}-U_{x y}$. Remind that $W_{\Delta}$ and $W_{x y}$ are curvature gradients obtainable from torsion balance measurements, while $U_{\Delta}$ and $U_{x y}$ are curvature gradients of the normal gravity field. $U_{\Delta}=10.26 \cos ^{2} \varphi$ and $U_{x y}=0$ referred to the GRS-80 in Eötvös Unit (1 Eötvös Unit $\left.=1 \mathrm{E}=10^{-9} \mathrm{~s}^{-2}\right)$, (Torge 1989). Now, by substituting (24) into (23):

$$
G_{i k}=\frac{n_{i k}}{4}\left[\left(\Delta W_{\Delta_{i}}+\Delta W_{\Delta_{k}}\right) \sin 2 \alpha_{i k}+\left(\Delta W_{x y i}+\Delta W_{x y k}\right) \cos 2 \alpha_{i k}\right]
$$

which, compared to (22) yields the basic equation, relating the variation of components of deflections of the vertical between two points to curvature gradients from torsion balance measurements:

$$
\begin{aligned}
& \left(\xi_{k}-\xi_{i}\right) \sin \alpha_{i k}-\left(\eta_{k}-\eta_{i}\right) \cos \alpha_{i k}= \\
& \quad \frac{n_{i k}}{4 g}\left[\left(\Delta W_{\Delta_{i}}+\Delta W_{\Delta_{k}}\right) \sin 2 \alpha_{i k}+\left(\Delta W_{x y_{i}}+\Delta W_{x y_{k}}\right) \cos 2 \alpha_{i k}\right] .
\end{aligned}
$$

Based on (26) there are $2 n$ unknowns in case of an arbitrary interpolation net of $n$ points. E.g. in case of a simple triangulation chain the $n$ points form $n$-2 triangles with $2 n-3$ triangle sides; so there are $2 n-3$ equations for the $2 n$ unknowns. For unambiguous solution further information are required (Völgyesi 1993, 1995). Two or more fixed points are needed where the deflections of the vertical are given.

\section{Computation of geoid heights from deflections of the vertical}

The basic principle of astronomical levelling gives us a definite mathematical relationship between geoid undulations and deflections of the vertical (Völgyesi 2001b). According to the notations of Fig. 3 we get

$$
d N=\vartheta d s
$$


where $\vartheta$ is the Pizzetti-type deflection of the vertical in the azimuth $\alpha$. Between any points $P_{i}$ and $P_{k}$ the geoid height change is

$$
\Delta N_{i k}=\int_{i}^{k} \vartheta(s) d s
$$

where $\vartheta(s)$ is the function of deflection of the vertical in the azimuth $\alpha_{i k}$, and $s$ is the distance between the two points (Biró 1985).

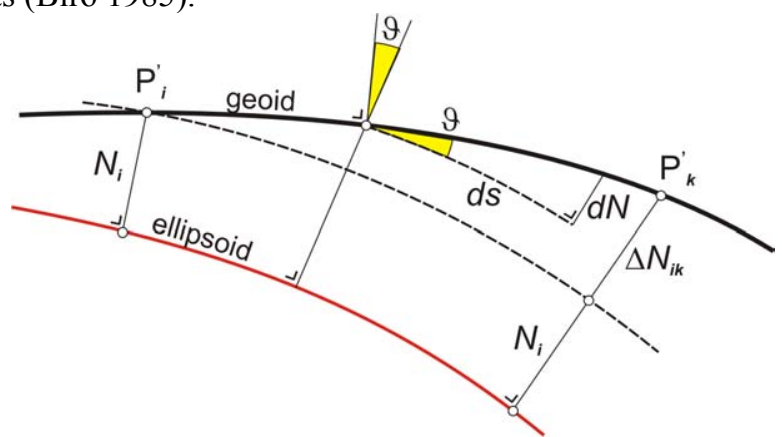

Fig. 3. Basic principle of astronomical levelling

If $P_{i}$ and $P_{k}$ are close together and $\vartheta(s)$ is a linear function between these points the integral (27) can be evaluated by a numerical integration (Völgyesi 1998, Tóth Gy, Völgyesi L 2002):

$$
N_{k}-N_{i}=\left(\frac{\xi_{i}+\xi_{k}}{2} \cos \alpha_{i k}+\frac{\eta_{i}+\eta_{k}}{2} \sin \alpha_{i k}\right) s_{i k}
$$

because of $\vartheta=\xi \cos \alpha+\eta \sin \alpha$. Using the original torsion balance points directly for the geoid computation instead of regular grid points is advantageous (Völgyesi 2001b). For an unambiguous solution one or more fixed points are needed where the initial geoid heights are given.

\section{Torsion balance measurements}

In the 20th century more than 60000 torsion balance measurements were made in Hungary (Szabó, 1999). Generally only the horizontal gradients of gravity $W_{z x}$ and $W_{z y}$ were developed for the purposes of geophysical prospecting, and the curvature values $W_{\Delta}$ and $W_{x y}$, which are very important for geodetic purposes, were left unprocessed. Unfortunately a small part of field books of torsion balance measurements were lost up to now, but the main part of measurements is still available (Völgyesi, 2001a). At present time serious efforts are going on for rescuing the historical torsion balance measurements, researchers of ELGI are creating the data of old field books to computer database. At present 24544 torsion balance measurements are available for further processing in computer database. Location of these torsion balance points can be seen on Fig. 4.

The name of the torsion balance station, measurement year, geographical latitude $\varphi$, geographical longitude $\lambda$, horizontal and curvature values $W_{z x}, W_{z y}, W_{\Delta}, 2 W_{x y}$ and terrain reductions of these gradients are stored in the database for each station.

Test computations were performed in an area extending over some $750 \mathrm{~km}^{2}$ (a rectangle shows the position of this test area in Fig. 4) and well measured by torsion balance, where both topographic conditions and the density of torsion balance measurements and astrogeodetic stations reflects average conditions in Hungary; and there was a possibility to check calculations because astrogeodetic and astrogravimetric data were available. In Figs. 5 and 6 curvature gradients $W_{\Delta}$ and $2 W_{x y}$ measured by torsion balance are visualized in the test area. 


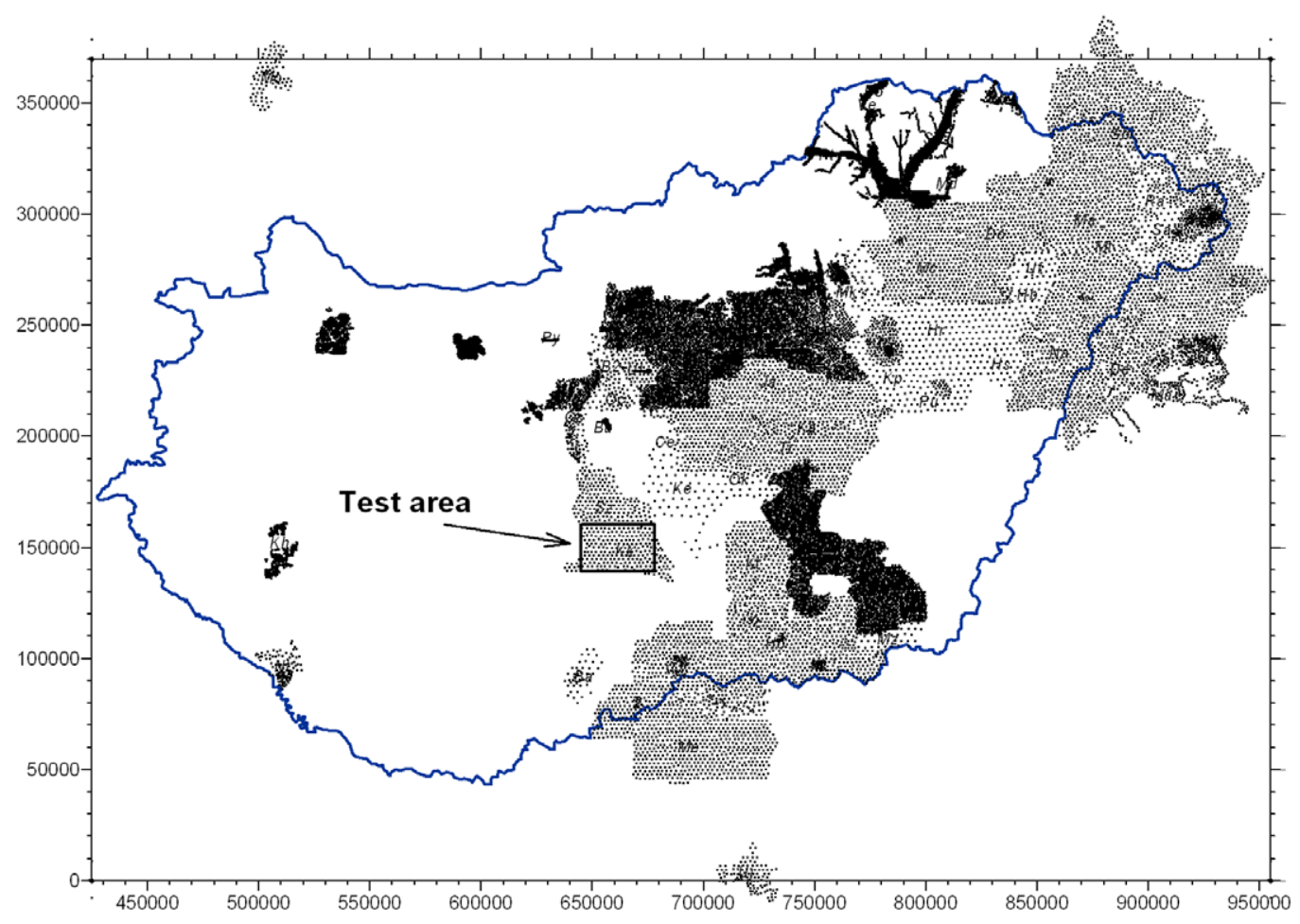

Fig. 4. Location of 24544 torsion balance points having been developed to computer database till now

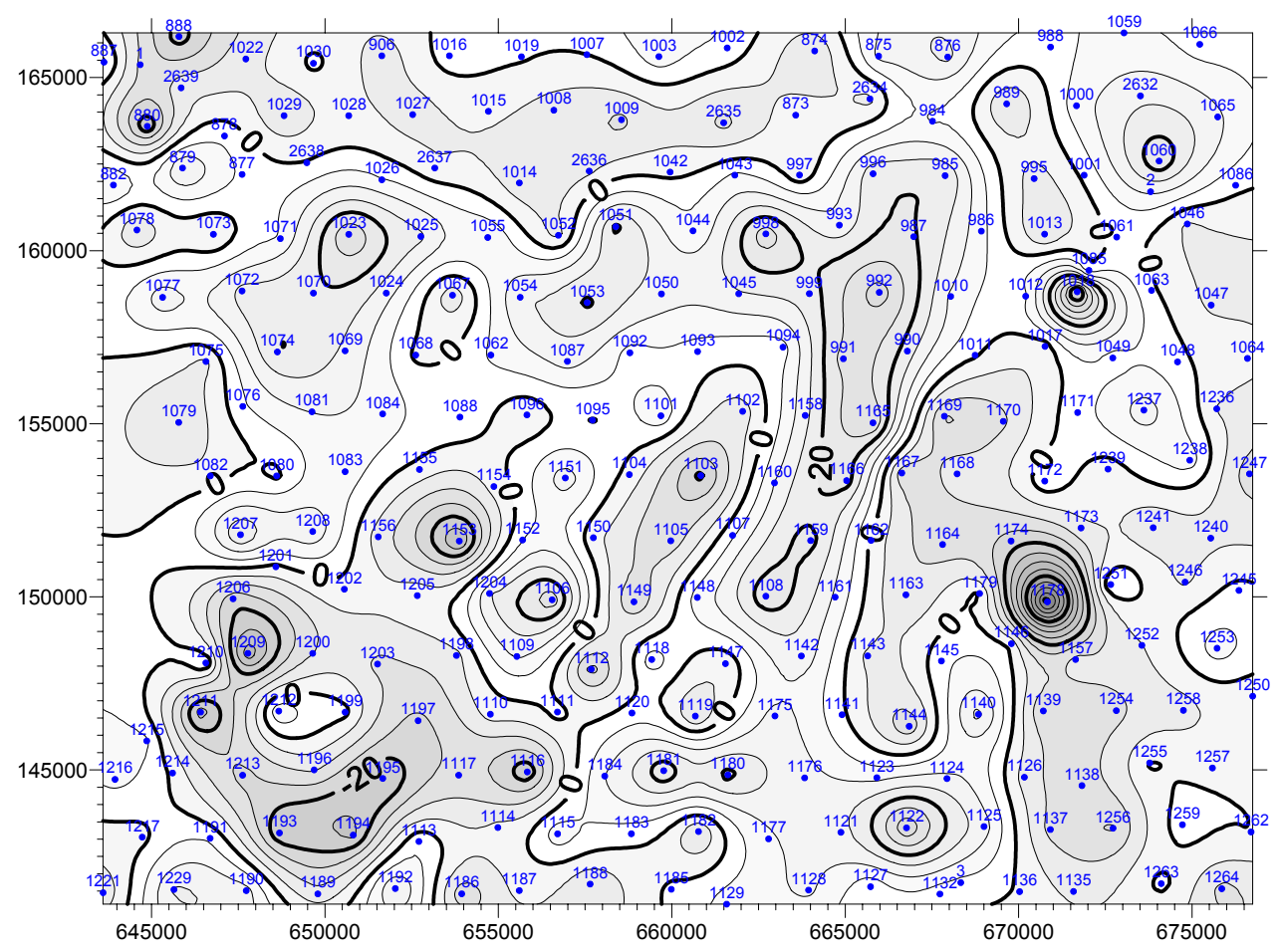

Fig. 5. Isoline map of curvature data $W_{\Delta}$ on the test area 


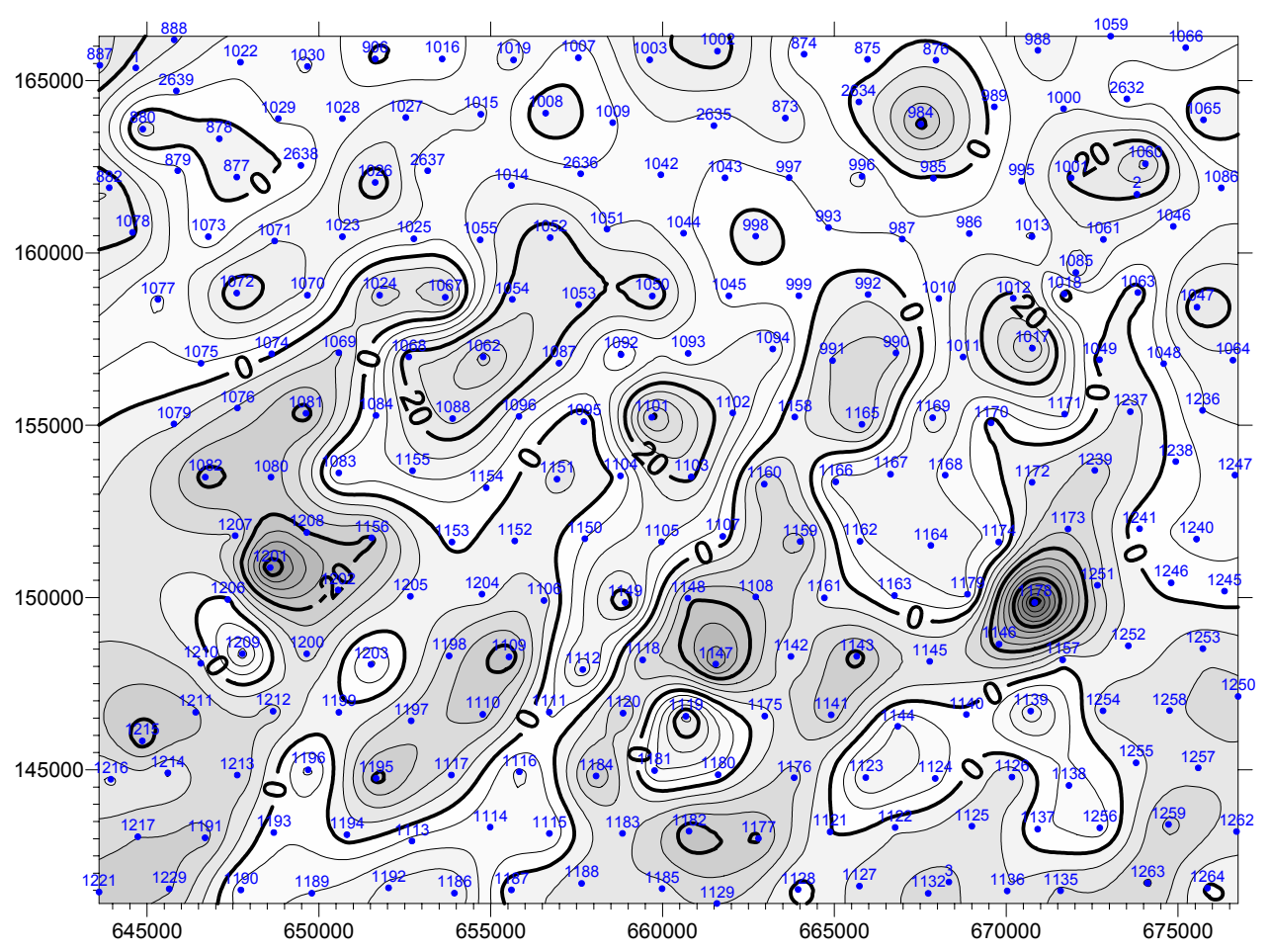

Fig. 6. Isoline map of curvature data $2 W_{x y}$ on the test area

\section{Test computations}

Software was developed for computations which can be used to determine deflections of the vertical and geoid undulations. Input-output window of this FuggoOrt software can be seen in Fig. 7.

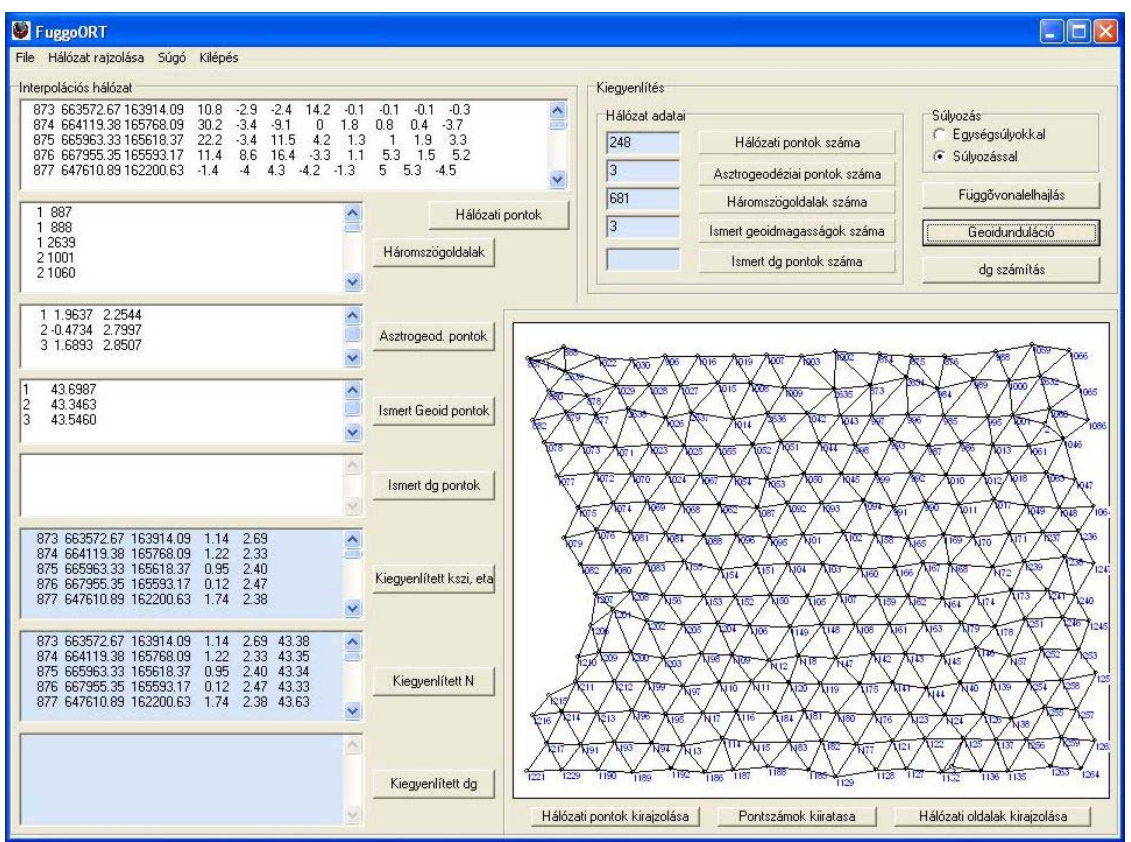

Fig. 7. Input-output window of the interpolation software 


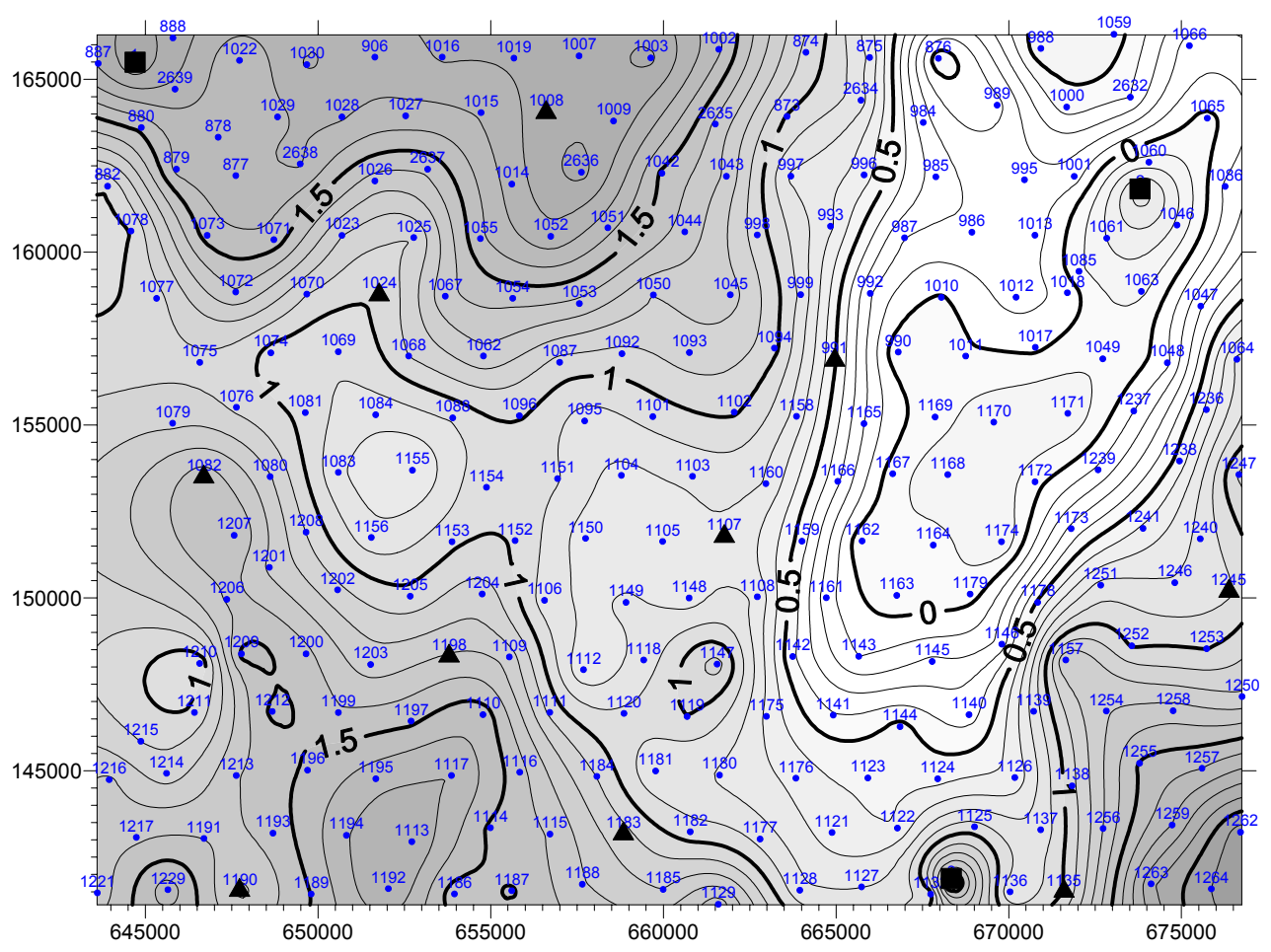

Fig. 8. Isoline map of $\xi$ component of interpolated deflections of the vertical

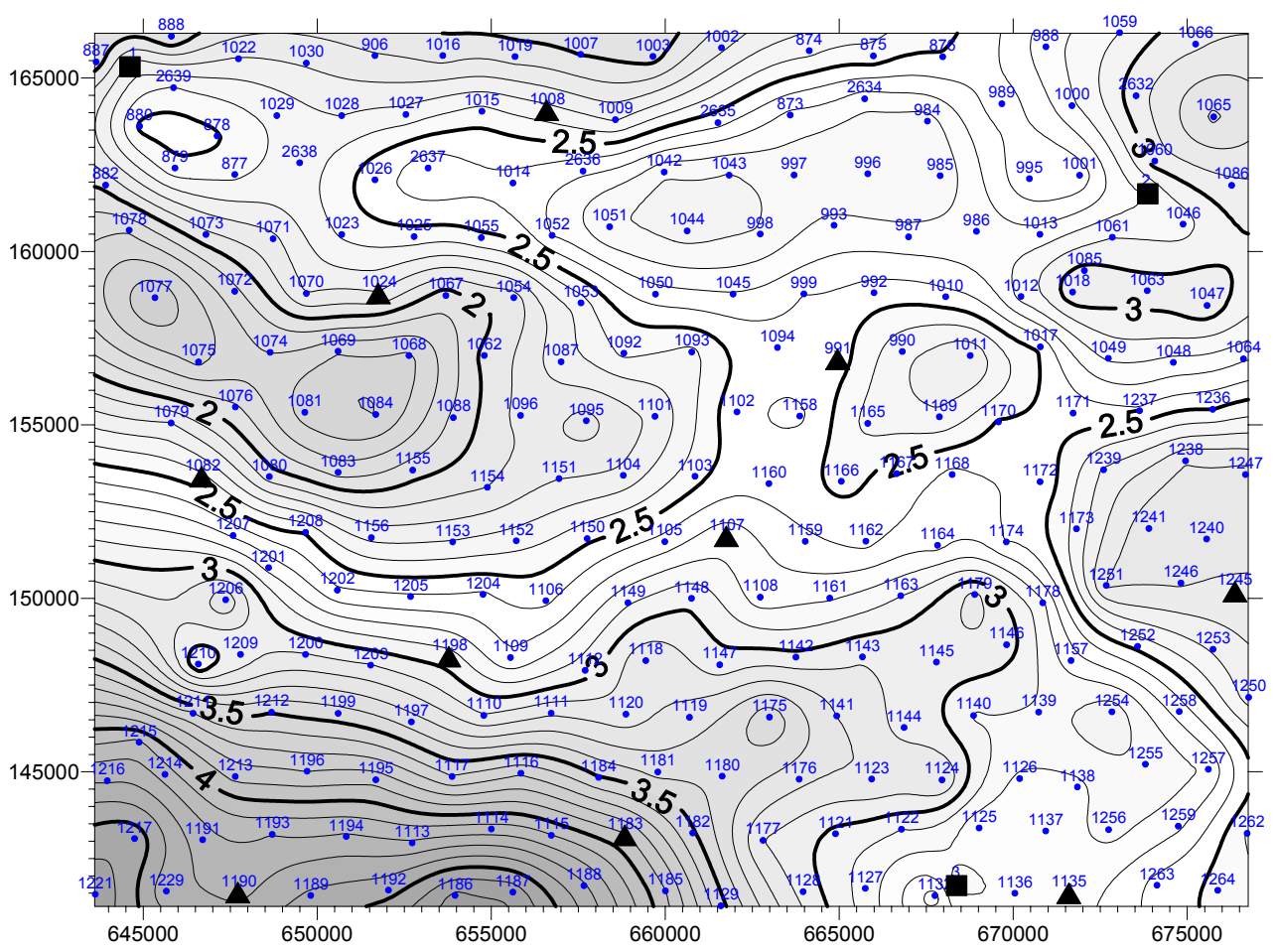

Fig. 9. Isoline map of $\eta$ component of interpolated deflections of the vertical 
Test computations were performed in the test area extending over about $750 \mathrm{~km}^{2}$. There are 248 torsion balance stations, and 13 points ( 3 astrogeodetic, and 10 astrogravimetric points) among them where $\xi, \eta$ and $N$ values are known referring to the GRS80 system. The 3 astrogeodetic points indicated with squares in Figs. 8, 9 and 10 were used as initial (fixed) points of interpolations and the 10 astrogravimetric points indicated with triangles in Figs. 8, 9 and 10 were used for checking of computations.

The interpolation network has 248 points in all and 245 of these are points with unknown deflections. Since there are two unknown components of deflection of the vertical at each point there are 498 unknowns for which 681 equations can be written.

Isoline maps of interpolated $\xi$ and $\eta$ components of deflections of the vertical can be seen in Figs. 8 and 9. Standard deviations computed at checkpoints are: $m_{\xi}= \pm 0.60^{\prime \prime}$ and $m_{\eta}= \pm 0.65^{\prime \prime}$.

Based on the computed deflection of the vertical components, using Eq (28) geoid computations were carried out. The deternined geoid map can be seen in Fig. 10. Geoid height differences computed at checkpoints are about $\pm 1-3 \mathrm{~cm}$.

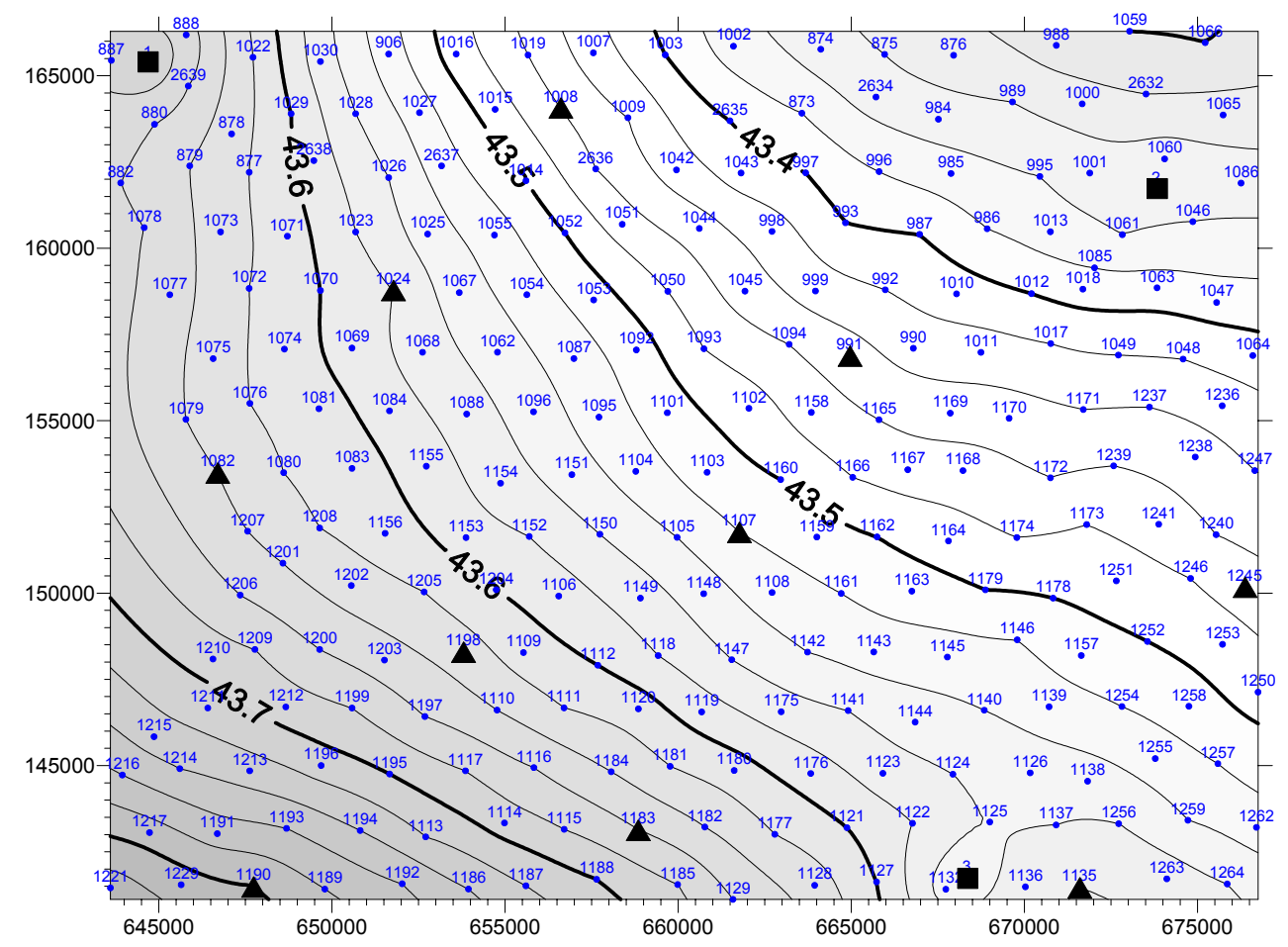

Fig. 10. Isoline map of geoid undulations

\section{Inversion reconstruction of gravity potential}

Further investigation is started for the inversion reconstruction of gravity potential based on torsion balance measurements. This method gives an additional possibility to determine deflections of the vertical based on torsion balance measurements. Based on the preliminary investigations accuracy of this method may be better than the accuracy of the former interpolation methods, and it is possible to avoid some types of earlier interpolation problems, like e.g. accuracy dependence of the geometrical configuration of the interpolation net (Völgyesi, 1995).

Based on the experimental computations by synthetic data, inversion algorithm seems to be remarkably stable (Dobróka, Völgyesi 2005). This method gives possibility to determine the potential 
function by a common inversion using both torsion balance measurements and deflections of the vertical data at a few given points. Many important features of the gravity field (e.g. deflections of the vertical at the unknown points) can be determined from this reconstructed potential function.

\section{Summary}

Our test computations confirm the fact that for large and more or less flat territories deflections of the vertical and geoid heights of acceptable accuracy can be computed from torsion balance measurements. Reliability of interpolated deflection of the vertical and geoid height values are about \pm 0.6 " and $\pm 1-3 \mathrm{~cm}$ respectively. In the next future waiting for the very precise measurements of the promising new modern gradiometers our method and software are ready for use to evaluate these further measurements.

\section{Acknowledgements.}

Our investigations are supported by the National Scientific Research Foundation (OTKA) grants T-037929.

\section{References}

Badekas J, Mueller I 1967: Reports of the Department of Geodetic Science, 98, The Ohio State University. Biró P 1985: Felsögeodézia, Tankönyvkiadó, Budapest

Dobróka M, Völgyesi L 2005: Geomatikai Közlemények VIII.

Magnitzki W A, W W Brovar (1964) Theorie der Figure der Erde. Veb Verlag, Berlin.

Selényi P 1953: Roland Eötvös Gesammelte Arbeiten. Akadémiai Kiadó, Budapest.

Szabó Z 1999: Magyar Geofizika. 40, 1, 26-38.

Torge W 1989: Gravimetry. Walter de Gruyter, Berlin New York.

Völgyesi L 1993: Periodica Polytechnica Civ.Eng. 37, 2, 137-166.

Völgyesi L 1995: Periodica Polytechnica Civ.Eng. 39, 1, 37-75.

Völgyesi L 1998: Reports of the Finnish Geodetic Institute 98:4, 145-151.

Völgyesi L 2001a: Reports on Geodesy, Warsaw University of Technology, 57, 2, 203-212.

Völgyesi L 2001b: Acta Geodaetica et Geophysica Hung. 36, 2, 153-162.

Tóth Gy, Völgyesi L 2002: Reports on Geodesy, Warsaw University of Technology, 61, 1, 171-182.

Völgyesi L (2005): Deflections of the vertical and geoid heights from gravity gradients. Acta Geodaetica et Geophysica Hungarica, Vol. 40, Nr. 2, pp. 147-159.

Dr. Lajos VÖLGYESI, Department of Geodesy and Surveying, Budapest University of Technology and Economics, H-1521 Budapest, Hungary, Müegyetem rkp. 3.

Web: http://sci.fgt.bme.hu/volgyesi E-mail: volgyesi@eik.bme.hu 